\title{
Midwall Fibrosis: Cardiac Magnetic Resonance Imaging in Risk Stratify- ing Cardiomyopathies
}

\author{
D.P. Ripley ${ }^{1,2, *}$, P. Garg ${ }^{3}$, A. Kotecha ${ }^{1}$, O.E. Gosling ${ }^{1,2}$ and N.G. Bellenger ${ }^{1,2}$ \\ ${ }^{I}$ Royal Devon \& Exeter NHS Foundation Trust, Barrack Road, Exeter, EX2 5DW, UK \\ ${ }^{2}$ Peninsula College of Medicine \& Dentistry, Barrack Road, Exeter, EX2 5DW, UK \\ ${ }^{3}$ Sheffield Teaching Hospital NHS Foundation Trust, Herries Road, Sheffield, South Yorks, S5 7AU, UK
}

\begin{abstract}
The United Kingdom's National Institute for Health and Care Excellence guidance on implantable cardiac defibrillator (ICD) therapy recommend ICD in those with left ventricular dysfunction and a high risk of sudden cardiac death (SCD). SCD accounts for 30\% deaths in non-ischaemic dilated cardiomyopathy (DCM), however risk stratifying and predicting SCD in DCM is a major management challenge. We present two cases demonstrating the potential role of cardiac magnetic resonance imaging in risk stratifying DCM.
\end{abstract}

Keywords: Magnetic Resonance Imaging, Sudden Cardiac Death, Ventricular Tachycardia, Dilated Cardiomyopathy, Fibrosis

\section{INTRODUCTION}

A 35 year old gentleman presented with haemodynamically unstable ventricular tachycardia (VT) which required DC cardioversion. Coronary angiography demonstrated normal epicardial vessels. Plain chest radiograph, serum ACE, $\mathrm{Ca}^{2+}$ and alpha-galactosidase were normal. Cardiac biopsy was not performed. Cardiac magnetic resonance (CMR) scan revealed extensive mid wall late gadolinium enhancement (LGE) and normal T2 weighted images (Fig 1). An implantable cardiac defibrillator (ICD) was inserted for secondary prevention.

A further patient presented with breathlessness (New York Heart Association Classification III) and palpitations. There was no history of syncope or family history of sudden cardiac death (SCD). Coronary angiography was normal. CMR demonstrated severe left ventricular systolic dysfunction (ejection fraction [EF] 21\%) with extensive mid wall LGE (Fig 2). Cardiac resynchronization therapy defibrillator (CRT-D) was implanted for primary prevention.

Case 1 illustrates an established example for the role of an ICD for secondary prevention whilst case 2 illustrates a potential role for CMR in risk stratification dilated cardiomyopathy for primary prevention. The United Kingdom's National Institute for Health and Care Excellence guidance on ICD implantation for primary prevention recommend ICD in those with left ventricular (LV) dysfunction (LV EF $\leq 35 \%$ ) in those with a high risk of SCD [1]. SCD accounts for $30 \%$ deaths in non-ischaemic dilated cardiomyopathy (DCM) and risk stratifying and predicting SCD in this group

*Address correspondence to this author at the Royal Devon \& Exeter NHS Foundation Trust, Barrack Road, Exeter, EX2 5DW, UK;

Tel: 01392411 611; Fax: 01392404 652; E-mail: david.ripley@nhs.net is a major management challenge. Although limited to two cases, we demonstrated how cardiac magnetic resonance may help in risk stratification prior to ICD implantation. Mid wall fibrosis has been shown to have prognostic value, predicting both VT and SCD [2, 3], however is not currently incorporated into any guideline for ICD implantation. Furthermore, current guidelines consider DCM as a homogenous entity and tissue characterisation by multi-parametric CMR can differentiate the cause.

\section{CONFLICT OF INTEREST}

The authors confirm that this article content has no conflict of interest.

\section{ACKNOWLEDGEMENTS}

DPR and OEG acknowledge the support of the Gawthorn Cardiac Trust

\section{LIST OF ABBREVIATIONS}

$\begin{array}{lll}\mathrm{ACE} & = & \text { Angiotensin Conventing Enzyme } \\ \mathrm{CMR} & = & \text { Cardiac Magnetic Resonance } \\ \mathrm{CRT}-\mathrm{D} & = & \text { Cardiac resynchronization therapy defibril- } \\ \text { lator }\end{array}$




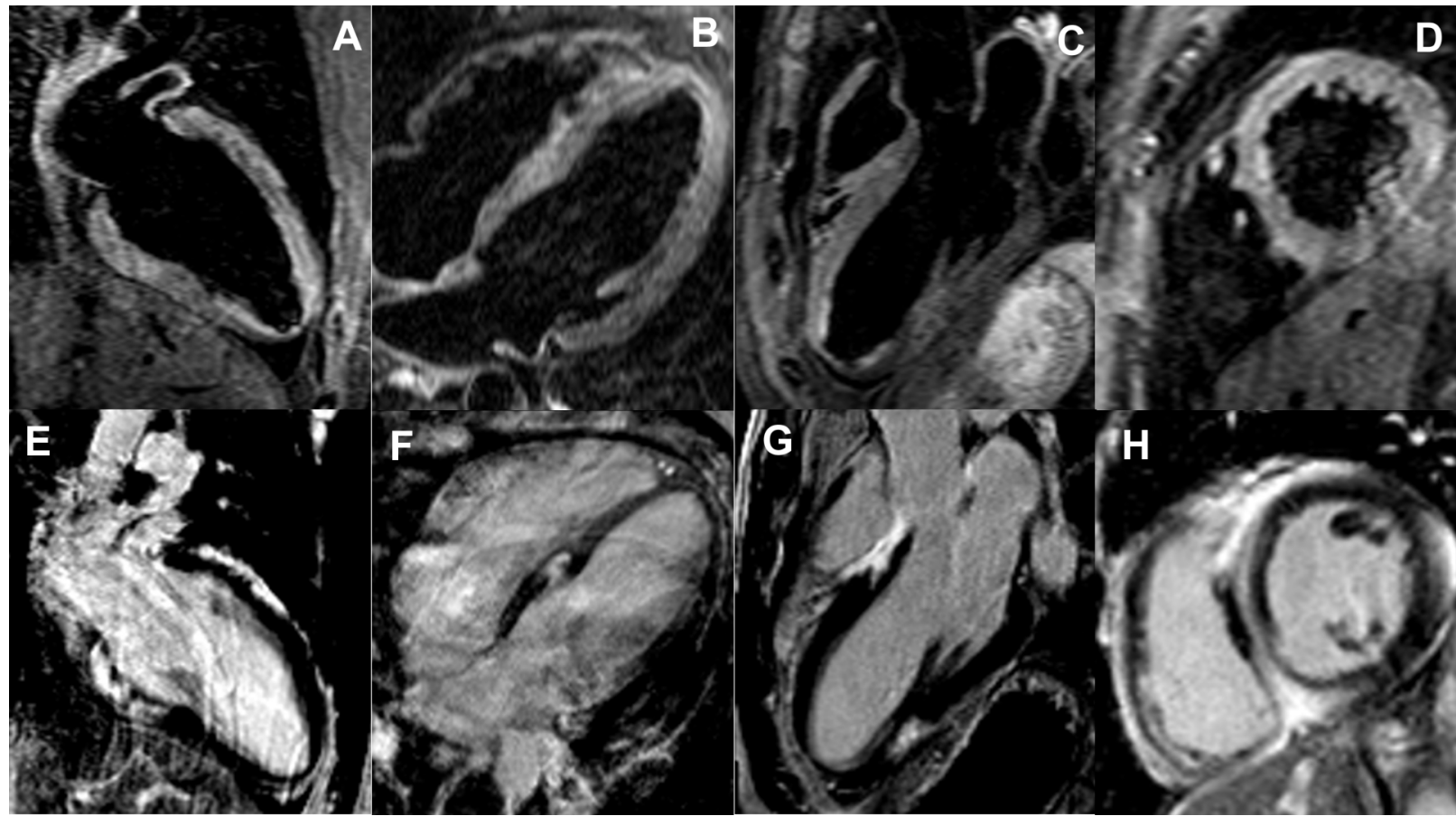

Fig. (1). Normal T2 imaging (A-D) and extensive mid myocardial wall late gadolinium enhancement (E-H) in the long and short axis views.

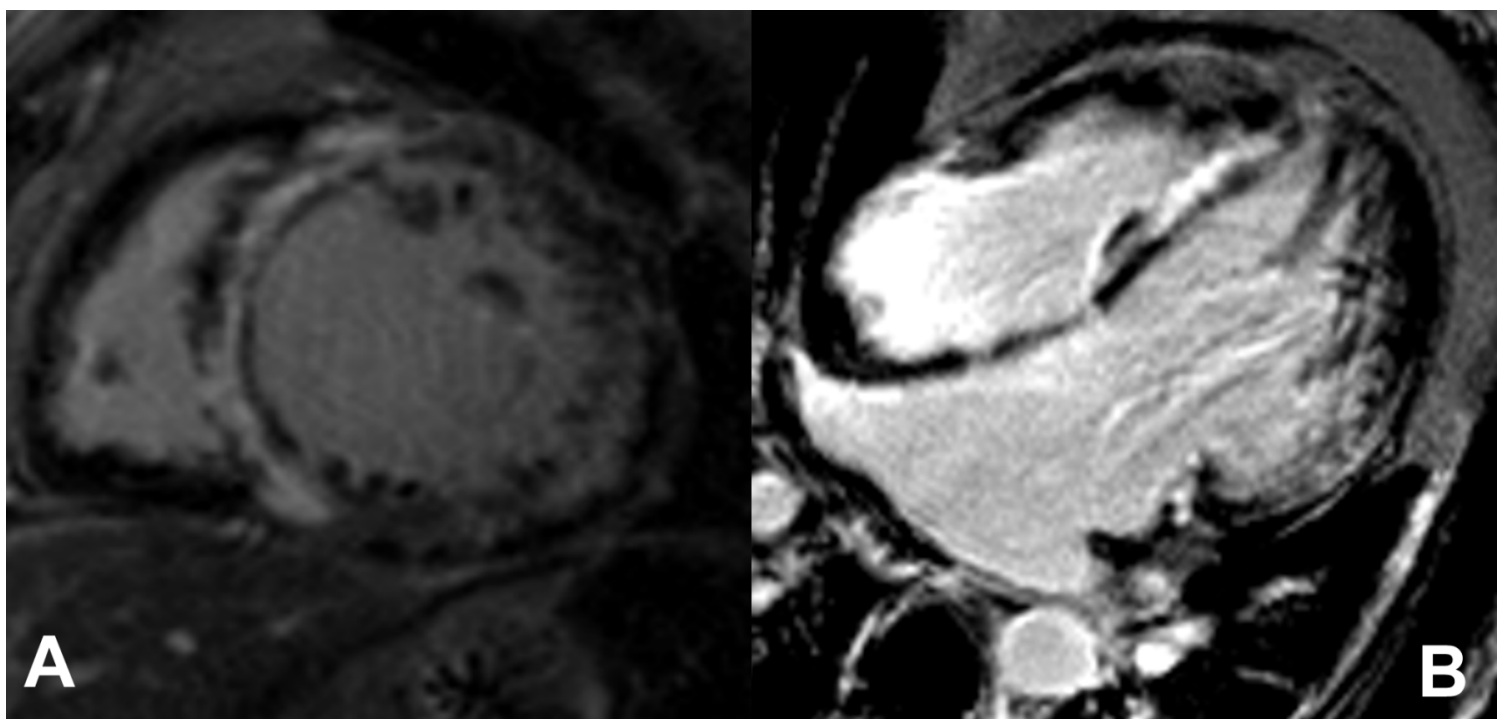

Fig. (2). Dilated cardiomyopathy with mid myocardial late gadolinium enhancement in short axis (A) and horizontal long axis (B) views.

\section{REFERENCES}

[1] NICE Technology Appraisal Guidance 314. Implantable cardioverter defibrillators and cardiac resynchronisation therapy for arrhythmias and heart failure. National Institue for Health and Care Excellence. June 2014.

[2] Assomull RG, Prasad SK, Lyne J, et al. Cardiovascular magnetic resonance, fibrosis, and prognosis in dilated cardiomyopathy. J Am Coll Cardiol 2006; 48: 1977-85.
[3] Lehrke S, Lossnitzer D, Schöb M, et al. Use of cardiovascular magnetic resonance for risk stratification in chronic heart failure: prognostic value of late gadolinium enhancement in patients with non-ischaemic dilated cardiomyopathy. Heart 2011; 97: 727-32.

(C) Ripley et al.; Licensee Bentham Open.

This is an open access article licensed under the terms of the Creative Commons Attribution Non-Commercial License (http://creativecommons.org/licenses/by$\mathrm{nc} / 3.0 /$ ), which permits unrestricted, non-commercial use, distribution and reproduction in any medium, provided the work is properly cited. 